International Mathematical Forum, Vol. 9, 2014, no. 3, 137 - 144

HIKARI Ltd, www.m-hikari.com

http://dx.doi.org/10.12988/imf.2014.312238

\title{
Exact Two Waves Solutions with Variable Amplitude to the KdV Equation ${ }^{1}$
}

\author{
Ying Huang \\ Department of Mathematics \\ Chuxiong Normal University \\ Chuxiong 675000, P.R. China
}

Copyright (C) 2014 Ying Huang. This is an open access article distributed under the Creative Commons Attribution License, which permits unrestricted use, distribution, and reproduction in any medium, provided the original work is properly cited.

\begin{abstract}
By means of the Darboux transformation method, a series of explicit variable amplitude-two-wave solutions to the Korteweg-de Vries (KdV) equation are generated from a particular solution with respect to the independent variable $\mathrm{x}$. Theses new solutions include single soliton solutions, two-soliton solutions, single periodic solutions, doubly periodic solutions.
\end{abstract}

Mathematics Subjection Classification: 35Q51; 35A

Keywords: KdV equation; Darboux transformation; soliton solution;periodic solution

\section{Introduction}

The KdV equation

$$
u_{t}+6 u u_{x}+u_{x x x}=0
$$

is a typical soliton equation, it's exact expressions of traveling wave solutions have been studied extensively in many papers $[1,2,3,4,5,6]$. Especially, the linetwo-soliton solutions obtained by the Inverse scattering method in [7] have been well known.

\footnotetext{
${ }^{1}$ This work was supported by the Chinese Natural Science Foundation Grant (11261001) and Yunnan Provincial Department of Education Research Foundation Grant (2012Y130).
} 
We know that, Darboux transformation method is one of the powerful and direct method for finding exact multiple line-soliton solutions, it is scarcely used for solving other type of multiple waves solutions because of the matter of calculation $[8,9,10,11,12]$. Generating new solutions from the non-trivial solution amounts to solving the variable coefficient partial differential system by this method, a major difficult is that for a given partial differential equations there is to date no completely systematic method of solving it, work has usually relied on some special techniques. In the paper, the author employ a exploratory method to solve partial differential system, further construct some two-wave solutions which amplitude depends on the independent variable $\mathrm{x}$.

\section{Solution to the lax pair}

As described in [13], the Lax pair for equation (1) is given by

$$
\left\{\begin{array}{l}
\Phi_{x}=\left(\begin{array}{cc}
0 & 1 \\
\lambda-u & 0
\end{array}\right) \Phi, \\
\Phi_{t}=\left(\begin{array}{cc}
u_{x} & -(4 \lambda+2 u) \\
-(4 \lambda+2 u)(\lambda-u)+u_{x x} & -u_{x}
\end{array}\right) \Phi
\end{array}\right.
$$

with the Darboux matrix

$$
D(x, t, \lambda)=\left(\begin{array}{cc}
-\sigma_{0} & 1 \\
\lambda-\lambda_{0}+\sigma_{0}^{2} & -\sigma_{0}
\end{array}\right),
$$

where $\lambda, \lambda_{0}$ are the spectral parameters, in particular, when $\Phi(x, t, \lambda)=$ $\left(a_{i j}(x, t, \lambda)\right)_{2 \times 2}$ is the fundamental solution matrix to the system (2) with $u=$ $u_{0}, \sigma_{0}$ is defined as

$$
\sigma_{0}=\frac{a_{21}\left(x, t, \lambda_{0}\right) \mu_{0}+a_{22}\left(x, t, \lambda_{0}\right) \gamma_{0}}{a_{11}\left(x, t, \lambda_{0}\right) \mu_{0}+a_{12}\left(x, t, \lambda_{0}\right) \gamma_{0}}
$$

here, $\mu_{0}$ and $\gamma_{0}$ are arbitrary constants, but $\mu_{0}^{2}+\gamma_{0}^{2} \neq 0$. Furthermore, if $u_{0}$ is a given solution to equation (1), then

$$
u_{1}=2 \lambda_{0}-u_{0}-2 \sigma_{0}^{2}
$$

becomes new solution for equation (1), which further concludes that

$$
u_{2}=2 \lambda_{1}-u_{1}-2 \sigma_{1}^{2}
$$

is another new solution based on $u_{1}$, where

$$
\sigma_{1}=\frac{\tilde{a}_{21}\left(x, t, \lambda_{1}\right) \mu_{1}+\tilde{a}_{22}\left(x, t, \lambda_{1}\right) \gamma_{1}}{\tilde{a}_{11}\left(x, t, \lambda_{1}\right) \mu_{1}+\tilde{a}_{12}\left(x, t, \lambda_{1}\right) \gamma_{1}},
$$


and $\tilde{\Phi}(x, t, \lambda)=D(x, t, \lambda) \Phi(x, t, \lambda)=\left(\tilde{a}_{i j}(x, t, \lambda)\right)_{2 \times 2}$ is the fundamental solution matrix of the Lax pair on $u_{1}, \mu_{1}, \gamma_{1}$ are similar to $\mu_{0}, \gamma_{0}$.

The essential procedure to construct new solutions is to solve the fundamental solution matrix of the lax pair on initial solution $u_{0}$. Substituting $u_{0}=-2 x^{-2}$ into the system (2) yields

$$
\left\{\begin{array}{l}
\Phi_{x}=\left(\begin{array}{cc}
0 & 1 \\
\lambda+2 x^{-2} & 0
\end{array}\right) \Phi, \\
\Phi_{t}=\left(\begin{array}{cc}
4 x^{-3} & -4 \lambda+4 x^{-2} \\
-4 \lambda^{2}-4 \lambda x^{-2}-4 x^{-4} & -4 x^{-3}
\end{array}\right) \Phi,
\end{array}\right.
$$

(8a) is an ordinary differential system with variable coefficient, we have no effective method to deal with it, as for (8b), when $x$ is looked as a parameter, it is an ordinary differential system with const coefficients. In the case of $\lambda>0$, with the eigenvalue method, we solve the characteristic equation

$$
\left|\begin{array}{cc}
\gamma-4 x^{-3} & 4 \lambda-4 x^{-2} \\
4 \lambda^{2}+4 \lambda x^{-2}+4 x^{-4} & \gamma+4 x^{-3}
\end{array}\right|=0
$$

and obtain the characteristic roots $\gamma_{1}=4 \lambda \sqrt{\lambda}, \gamma_{2}=-4 \lambda \sqrt{\lambda}$. After calculating the characteristic vectors associated with $\gamma_{1}$ and $\gamma_{2}$, we obtain two basic solutions to $(8 b)$

$$
\left(\begin{array}{c}
\left(\lambda-x^{-2}\right) e^{4 \lambda \sqrt{\lambda} t} \\
\left(x^{-3}-\lambda \sqrt{\lambda}\right) e^{4 \lambda \sqrt{\lambda} t}
\end{array}\right),\left(\begin{array}{c}
\left(\lambda-x^{-2}\right) e^{-4 \lambda \sqrt{\lambda} t} \\
\left(x^{-3}+\lambda \sqrt{\lambda}\right) e^{-4 \lambda \sqrt{\lambda} t}
\end{array}\right) .
$$

Of course,

$$
k_{1}(x)\left(\begin{array}{c}
\left(\lambda-x^{-2}\right) e^{4 \lambda \sqrt{\lambda} t} \\
\left(x^{-3}-\lambda \sqrt{\lambda}\right) e^{4 \lambda \sqrt{\lambda} t}
\end{array}\right)
$$

is also solution to $(8 \mathrm{~b})$, where $k_{1}(x)$ is function to be determined later. In order to probe whether it is a solution of (8a), substituting it into (8a), we have

$$
\begin{gathered}
k_{1}^{\prime}(x)\left(x^{-2}-\lambda\right)=k_{1}(x)\left(\lambda \sqrt{\lambda}+x^{-3}\right) \\
k_{1}^{\prime}(x)\left(\lambda \sqrt{\lambda}-x^{-3}\right)=-k_{1}(x)\left(\lambda^{2}+\lambda x^{-2}+x^{-4}\right),
\end{gathered}
$$

more interesting, (9a) and (9b) have the same solution $k_{1}(x)=\frac{x}{1-\sqrt{\lambda} x} e^{-\sqrt{\lambda} x}$. In parallel, we know

$$
k_{2}(x)\left(\begin{array}{c}
\left(\lambda-x^{-2}\right) e^{4 \lambda \sqrt{\lambda} t} \\
\left(x^{-3}-\lambda \sqrt{\lambda}\right) e^{4 \lambda \sqrt{\lambda} t}
\end{array}\right)
$$


is also solution to $(8 \mathrm{~b})$, where $k_{2}(x)=\frac{x}{1+\sqrt{\lambda} x} e^{\sqrt{\lambda} x}$. In short, we obtain the fundamental solution matrix to the system $(8)$

$$
\Phi(x, t, \lambda)=\left(\begin{array}{cc}
-\left(\sqrt{\lambda}+x^{-1}\right) e^{\xi} & \left(\sqrt{\lambda}-x^{-1}\right) e^{-\xi} \\
\left(\lambda+\sqrt{\lambda} x^{-1}+x^{-2}\right) e^{\xi} & \left(\lambda-\sqrt{\lambda} x^{-1}+x^{-2}\right) e^{-\xi}
\end{array}\right),
$$

where $\xi=\sqrt{\lambda}(4 \lambda t-x)$.

When $\lambda<0$, the fundamental solution matrix (10) is complex, that is to say, the system (8) has two linearly independent complex solutions. We know that the real and imaginary parts of a complex-valued solution are also solutions, thus taking

$$
\Phi^{\prime}(x, t, \lambda)=\left(\begin{array}{cc}
\sqrt{-\lambda} \sin \theta-x^{-1} \cos \theta & -\left(\sqrt{-\lambda} \cos \theta+x^{-1} \sin \theta\right) \\
I & J
\end{array}\right)
$$

as the fundamental solution matrix to the system (8), where $I=\lambda \cos \theta-$ $x^{-1}\left(\sqrt{-\lambda} \sin \theta-x^{-1} \cos \theta\right), J=\lambda \sin \theta+x^{-1}\left(\sqrt{-\lambda} \cos \theta+x^{-1} \sin \theta\right), \theta=\sqrt{-\lambda}(4 \lambda t-$ $x)$.

Similarly, we obtain the fundamental solution matrix to the system (8) for the case of $\lambda=0$

$$
\Phi^{\prime \prime}(x, t, 0)=\left(\begin{array}{cc}
x^{2}+12 t x^{-1} & x^{-1} \\
2 x-12 t x^{-2} & -x^{-2}
\end{array}\right) .
$$

\section{Solutions to the $\mathrm{KdV}$ equation}

Substituting the entries of (10) into (4) shows

$$
\sigma_{0}=-x^{-1}-\frac{\lambda_{0} x\left(e^{\xi_{0}} \mu_{0}+e^{-\xi_{0}} \gamma_{0}\right)}{e^{\xi_{0}} \mu_{0}+e^{-\xi_{0}} \gamma_{0}+\sqrt{\lambda_{0}} x\left(e^{\xi_{0}} \mu_{0}-e^{-\xi_{0}} \gamma_{0}\right)}
$$

with $\xi_{0}=\xi\left(\lambda_{0}\right)$. Then substituting (11) into (5) yields

$$
u_{1}=\frac{-2 \lambda_{0}\left[\left(e^{\xi_{0}} \mu_{0}+e^{-\xi_{0}} \gamma_{0}\right)^{2}+4 \lambda_{0} \mu_{0} \gamma_{0} x^{2}\right]}{\left[e^{\xi_{0}} \mu_{0}+e^{-\xi_{0}} \gamma_{0}+\sqrt{\lambda_{0}} x\left(e^{\xi_{0}} \mu_{0}-e^{-\xi_{0}} \gamma_{0}\right)\right]^{2}}
$$

in (12), choosing $\mu_{0}=e^{c_{0}}, \gamma_{0}=e^{-c_{0}}$ and $\mu_{0}=e^{c_{0}}, \gamma_{0}=-e^{-c_{0}}$ respectively, we have new soliton solutions

$$
u_{1-1}=\frac{-2 \lambda_{0}\left(\cosh ^{2} \eta_{0}+\lambda_{0} x^{2}\right)}{\left(\cosh \eta_{0}+\sqrt{\lambda_{0}} x \sinh \eta_{0}\right)^{2}}
$$

and

$$
u_{1-2}=\frac{-2 \lambda_{0}\left(\sinh ^{2} \eta_{0}-\lambda_{0} x^{2}\right)}{\left(\sinh \eta_{0}+\sqrt{\lambda_{0}} x \cosh \eta_{0}\right)^{2}}
$$


respectively, where $c_{0}$ is an arbitrary constant, $\eta_{0}=\xi_{0}+c_{0}$.

Now we construct the variable amplitude-two-soliton solutions to the KdV equation generated from $u_{1}$. For convenience, we first give the new solution which is expressed in terms of $\sigma_{0}$ rather than $u_{1}$, then consider $\mu_{0}$ and $\gamma_{0}$ in $\sigma_{0}$. According to the Darboux matrix (3) and (10), we can obtain the fundamental matrix to the lax pair associated with the known solution $u_{1}$ in the following manner

$$
\tilde{\Phi}(x, t, \lambda)=\left(\begin{array}{cc}
-\sigma_{0} & 1 \\
\lambda-\lambda_{0}+\sigma_{0}^{2} & -\sigma_{0}
\end{array}\right) \Phi(x, t, \lambda)=\left(\begin{array}{cc}
P & Q \\
S & T
\end{array}\right),
$$

where $P=\left[\lambda+\left(\sigma_{0}+x^{-1}\right)\left(\sqrt{\lambda}+x^{-1}\right)\right] e^{\xi}, Q=\left[\lambda-\left(\sigma_{0}+x^{-1}\right)\left(\sqrt{\lambda}-x^{-1}\right)\right] e^{-\xi}$ $S=-\left[\lambda \sigma_{0}+\left(\lambda-\lambda_{0}+\sigma_{0}^{2}+\sigma_{0} x^{-1}\right)\left(\sqrt{\lambda}+x^{-1}\right)\right] e^{\xi}, T=\left[-\lambda \sigma_{0}+\left(\lambda-\lambda_{0}+\sigma_{0}^{2}+\right.\right.$ $\left.\left.\sigma_{0} x^{-1}\right)\left(\sqrt{\lambda}-x^{-1}\right)\right] e^{-\xi}$, from $(7)$, this implies

$$
\begin{aligned}
& \sigma_{1}=-\sigma_{0}- \\
& \frac{\left(\lambda_{1}-\lambda_{0}\right)\left[\left(e^{\xi_{1}} \mu_{1}+e^{-\xi_{1}} \gamma_{1}\right)+\sqrt{\lambda_{1}} x\left(e^{\xi_{1}} \mu_{1}-e^{-\xi_{1}} \gamma_{1}\right)\right]}{\lambda_{1} x\left(e^{\xi_{1}} \mu_{1}+e^{-\xi_{1}} \gamma_{1}\right)+\left(\sigma_{0}+x^{-1}\right)\left[\left(e^{\xi_{1}} \mu_{1}+e^{-\xi_{1}} \gamma_{1}\right)+\sqrt{\lambda_{1}} x\left(e^{\xi_{1}} \mu_{1}-e^{-\xi_{1}} \gamma_{1}\right)\right]},
\end{aligned}
$$

where $\xi_{1}=\xi\left(\lambda_{1}\right)$. Choosing $\mu_{1}=e^{c_{1}}, \gamma_{1}=e^{-c_{1}}$ and $\mu_{1}=e^{c_{1}}, \gamma_{1}=-e^{-c_{1}}$ in the above formulation, respectively, we obtain

$$
\sigma_{11}=-\sigma_{0}-\frac{\left(\lambda_{1}-\lambda_{0}\right)\left(\cosh \xi_{1}+\sqrt{\lambda_{1}} x \sinh \xi_{1}\right)}{\lambda_{1} x \cosh \xi_{1}+\left(\sigma_{0}+x^{-1}\right)\left(\cosh \xi_{1}+\sqrt{\lambda_{1}} x \sinh \xi_{1}\right)},
$$

and

$$
\sigma_{12}=-\sigma_{0}-\frac{\left(\lambda_{1}-\lambda_{0}\right)\left(\sinh \xi_{1}+\sqrt{\lambda_{1}} x \cosh \xi_{1}\right)}{\lambda_{1} x \sinh \xi_{1}+\left(\sigma_{0}+x^{-1}\right)\left(\sinh \xi_{1}+\sqrt{\lambda_{1}} x \cosh \xi_{1}\right)},
$$

Substituting (5) and (15) into (6), we get

$$
\begin{aligned}
u_{2}= & -2 x^{-2}+2\left(\lambda_{1}-\lambda_{0}\right) \times \\
& \frac{\lambda_{1}\left(\cosh ^{2} \eta_{1}+\lambda_{1} x^{2}\right)+\left(\lambda_{0}-\sigma_{0}^{2}+x^{-2}\right)\left(\cosh \eta_{1}+\sqrt{\lambda_{1}} x \sinh \eta_{1}\right)^{2}}{\left[\lambda_{1} x \cosh \eta_{1}+\left(\sigma_{0}+x^{-1}\right)\left(\cosh \eta_{1}+\sqrt{\lambda_{1}} x \sinh \eta_{1}\right)\right]^{2}} .
\end{aligned}
$$

where $\eta_{1}=\xi\left(\lambda_{1}\right)+c_{1}, c_{1}$ is an arbitrary constant. Substituting (13) into (17), at the same time, choosing $\mu_{0}=e^{c_{0}}, \gamma_{0}=e^{-c_{0}}$ and $\mu_{0}=e^{c_{0}}, \gamma_{0}=-e^{-c_{0}}$, respectively, we obtain the two-soliton solutions

$$
u_{2-1}=2 \frac{\left(\lambda_{1}-\lambda_{0}\right) x^{2}\left(\lambda_{1}^{2} A_{1}^{2}-\lambda_{0}^{2} B_{1}^{2}\right)-\lambda_{0} \lambda_{1}\left(\cosh \eta_{1} A_{1}-\cosh \eta_{0} B_{1}\right)^{2}}{x^{2}\left(\lambda_{1} \cosh \eta_{1} A_{1}-\lambda_{0} \cosh \eta_{0} B_{1}\right)^{2}}
$$

and

$$
u_{2-2}=2 \frac{\left(\lambda_{1}-\lambda_{0}\right) x^{2}\left(\lambda_{1}^{2} A_{2}^{2}+\lambda_{0}^{2} B_{1}^{2}\right)-\lambda_{0} \lambda_{1}\left(\cosh \eta_{1} A_{2}-\sinh \eta_{0} B_{1}\right)^{2}}{x^{2}\left(\lambda_{1} \cosh \eta_{1} A_{2}-\lambda_{0} \sinh \eta_{0} B_{1}\right)^{2}}
$$


respectively, where $A_{1}=\cosh \eta_{0}+\sqrt{\lambda_{0}} x \sinh \eta_{0}, B_{1}=\cosh \eta_{1}+\sqrt{\lambda_{1}} x \sinh \eta_{1}$, $A_{2}=\sinh \eta_{0}+\sqrt{\lambda_{0}} x \cosh \eta_{0}$.

In the same way, compound (5), (6), (13) and (16), we obtain new solutions

$$
u_{2-3}=2 \frac{\left(\lambda_{0}-\lambda_{1}\right) x^{2}\left(\lambda_{1}^{2} A_{1}^{2}+\lambda_{0}^{2} B_{2}^{2}\right)-\lambda_{0} \lambda_{1}\left(\sinh \eta_{1} A_{1}-\cosh \eta_{0} B_{2}\right)^{2}}{x^{2}\left(\lambda_{1} \sinh \eta_{1} A_{1}-\lambda_{0} \cosh \eta_{0} B_{2}\right)^{2}}
$$

and

$$
u_{2-4}=2 \frac{\left(\lambda_{1}-\lambda_{0}\right) x^{2}\left(\lambda_{0}^{2} B_{2}^{2}-\lambda_{1}^{2} A_{2}^{2}\right)-\lambda_{0} \lambda_{1}\left(\sinh \eta_{1} A_{2}-\sinh \eta_{0} B_{2}\right)^{2}}{x^{2}\left(\lambda_{1} \sinh \eta_{1} A_{2}-\lambda_{0} \sinh \eta_{0} B_{2}\right)^{2}}
$$

with $B_{2}=\sinh \eta_{1}+\sqrt{\lambda_{1}} x \cosh \eta_{1}$.

Next, the same calculation as the case $\lambda>0$ can be done in $\lambda<0$, combining (11) with (4), we obtain

$$
u_{1-1}^{\prime}=\frac{-2 \lambda_{0}\left(\cos ^{2} \theta_{0}+\lambda_{0} x^{2}\right)}{\left(\cos \theta_{0}-\sqrt{-\lambda_{0}} x \sin \theta_{0}\right)^{2}}
$$

and

$$
u_{1-2}^{\prime}=\frac{-2 \lambda_{0}\left(\sin ^{2} \theta_{0}+\lambda_{0} x^{2}\right)}{\left(\sin \theta_{0}+\sqrt{-\lambda_{0}} x \cos \theta_{0}\right)^{2}}
$$

with $\theta_{0}=\theta\left(\lambda_{0}\right)$. In exactly the same manner, we further obtain new doubly periodic solutions

$$
\begin{aligned}
& u_{2-1}^{\prime}=2 \frac{\left(\lambda_{1}-\lambda_{0}\right) x^{2}\left(\lambda_{1}^{2} A_{1}^{\prime 2}-\lambda_{0}^{2} B_{1}^{\prime 2}\right)-\lambda_{0} \lambda_{1}\left(\cos \theta_{1} A_{1}^{\prime}-\cos \theta_{0} B_{1}^{\prime}\right)^{2}}{x^{2}\left(\lambda_{1} \cos \theta_{1} A_{1}^{\prime}-\lambda_{0} \cos \theta_{0} B_{1}^{\prime}\right)^{2}}, \\
& u_{2-2}^{\prime}=2 \frac{\left(\lambda_{1}-\lambda_{0}\right) x^{2}\left(\lambda_{1}^{2} A_{2}^{\prime 2}-\lambda_{0}^{2} B_{1}^{\prime 2}\right)-\lambda_{0} \lambda_{1}\left(\cos \theta_{1} A_{2}^{\prime}-\sin \theta_{0} B_{1}^{\prime}\right)^{2}}{x^{2}\left(\lambda_{1} \cos \theta_{1} A_{2}^{\prime}-\lambda_{0} \sin \theta_{0} B_{1}^{\prime}\right)^{2}} \\
& u_{2-3}^{\prime}=2 \frac{\left(\lambda_{1}-\lambda_{0}\right) x^{2}\left(\lambda_{1}^{2} A_{1}^{\prime 2}-\lambda_{0}^{2} B_{2}^{\prime 2}\right)-\lambda_{0} \lambda_{1}\left(\sin \theta_{1} A_{1}^{\prime}-\cos \theta_{0} B_{2}^{\prime}\right)^{2}}{x^{2}\left(\lambda_{1} \sin \theta_{1} A_{1}^{\prime}-\lambda_{0} \cos \theta_{0} B_{2}^{\prime}\right)^{2}}
\end{aligned}
$$

together with

$$
u_{2-4}^{\prime}=2 \frac{\left(\lambda_{1}-\lambda_{0}\right) x^{2}\left(\lambda_{1}^{2} A_{2}^{\prime 2}-\lambda_{0}^{2} B_{2}^{\prime 2}\right)-\lambda_{0} \lambda_{1}\left(\sin \theta_{1} A_{2}^{\prime}-\sin \theta_{0} B_{2}^{\prime}\right)^{2}}{x^{2}\left(\lambda_{1} \sin \theta_{1} A_{2}^{\prime}-\lambda_{0} \sin \theta_{0} B_{2}^{\prime}\right)^{2}},
$$

where $A_{1}^{\prime}=\cos \theta_{0}-\sqrt{-\lambda_{0}} x \sin \theta_{0}, B_{1}^{\prime}=\cos \theta_{1}-\sqrt{-\lambda_{1}} x \sin \theta_{1}, A_{2}^{\prime}=\sin \theta_{0}+$ $\sqrt{-\lambda_{0}} x \cos \theta_{0}, B_{2}^{\prime}=\sin \theta_{1}+\sqrt{-\lambda_{1}} x \cos \theta_{1}$. 
Finally, from (4) and (12), we get nonlinear rational solution

$$
u^{\prime \prime}=\frac{8 \mu_{0} x\left(12 \mu_{0} t+\gamma_{0}\right)}{\left(\mu_{0} x^{3}+12 \mu_{0} t+\gamma_{0}\right)^{2}} .
$$

\section{References}

[1] J. L. Varez, A.Durán, Error propagation when approximating multi-solitons:The $\mathrm{KdV}$ equation with as a case study, Applied Mathematics and Computation, 217 (2010), 1522-1539.

[2] J. 1. Yin, L.X. Tian, Classification of the traveling waves in the nonlinear dispersive KdV equation. Nonlinear Analysis, 73(2010),465-470.

[3] A. Biswas, M. D. Petkovic, D. Milovic, Topological and non- Topological exact soliton solution of the KdV equation. Commun Nolnear Sci Numer Simulat, 15(2010),3263-3269.

[4] M. Nivala, B. Deconinck. Periodic finite-genus solutions of the KdV equation are orbitally stable. Physic D, 239(2011),1147-1158.

[5] Y.Yamamoto, T. Nagase, M.Ohmiya, Appell's lemma and conservation laws of KdV equation,Journal of Computational and Applied Mathematics, 233(2010),16121618.

[6] N. K. Ameine, M. A. Ramadau, A small time solutions for the KdV equation using Bubnov-Galerkin finite element method. Journal of the Egyptian Mathematical Society, 9(2011),118-123.

[7] M.J. Ablowitz , P. A. Clarkson, Solitons, Nonlinear Evolutions and Inverse Scattering. Cambridge University Press, 1991, 23-99.

[8] X. M Li, A. H. Chen, Darboux transformation and multi-soliton solutions of Boussinesq-Burgers equation Physics Letters A, 342(2005),413-420.

[9] Y. Wang, L. J. Shen, Dianlou Du.Darboux transformation and explicit solutions for some (2+1)-dimensional equation. Physics Letters A, 366(2007),230240 .

[10] H. X. Wu, Y. B. Zeng, T. Y. Fan, Complexitions of the modified KdV equation by Darboux transformation. Applied Mathematics and Computation, 196(2008), 501-510.

[11] H. A. Zedan, Exact solutions for the generalized KdV equation by using Backlund transformation, Journal of the Franklin institute,348(2011)1751-1768.

[12] Y. Huang, New no-traveling wave solutions for the Liouville equation by Bäcklund transformation method,Nonlinear Dynamics 72(2013),87-90. 
[13] C.H. Gu, H.S. Hu, Z.X. Zhou, Darboux Transformation In Soliton Theory And Its Applications On Geometry. Shanghai Scientific and Technical Publishers, China, 2005.

Received: December 7, 2013 